\title{
ARTICLE
}

Received 18 Jul 2015 | Accepted 16 Nov 2015 | Published 17 Dec 2015

DOI: $10.1038 /$ ncomms10223

OPEN

\section{ATP hydrolysis assists phosphate release and promotes reaction ordering in $\mathrm{F}_{1}$-ATPase}

Chun-Biu Li ${ }^{1}$, Hiroshi Ueno ${ }^{2}$, Rikiya Watanabe $2,3,4$, Hiroyuki Noji ${ }^{2,4}$ \& Tamiki Komatsuzaki ${ }^{1}$

$F_{1}$-ATPase $\left(F_{1}\right)$ is a rotary motor protein that can efficiently convert chemical energy to mechanical work of rotation via fine coordination of its conformational motions and reaction sequences. Compared with reactant binding and product release, the ATP hydrolysis has relatively little contributions to the torque and chemical energy generation. To scrutinize possible roles of ATP hydrolysis, we investigate the detailed statistics of the catalytic dwells from high-speed single wild-type $F_{1}$ observations. Here we report a small rotation during the catalytic dwell triggered by the ATP hydrolysis that is indiscernible in previous studies. Moreover, we find in freely rotating $F_{1}$ that ATP hydrolysis is followed by the release of inorganic phosphate with low synthesis rates. Finally, we propose functional roles of the ATP hydrolysis as a key to kinetically unlock the subsequent phosphate release and promote the correct reaction ordering.

\footnotetext{
${ }^{1}$ Research Institute for Electronic Science, Hokkaido University, Sapporo 001-0020, Japan. ${ }^{2}$ Department of Applied Chemistry, Graduate School of Engineering, University of Tokyo, Tokyo 113-8656, Japan. ${ }^{3}$ PRESTO, Japan Science and Technology Agency, Tokyo 113-8656, Japan. ${ }^{4}$ CREST, Japan Science and Technology Agency, Tokyo 113-8656, Japan. Correspondence and requests for materials should be addressed to T.K. (email: tamiki@es.hokudai.ac.jp).
} 
T he rotary protein motor $\mathrm{F}_{1}$-ATPase $\left(\mathrm{F}_{1}\right)$ with subunits $\alpha_{3} \beta_{3} \gamma \delta \varepsilon$ is a catalytic sub-complex of the $\mathrm{F}_{\mathrm{o}} \mathrm{F}_{1}$-ATP synthase that catalyses the synthesis of ATP from ADP and inorganic phosphate $\left(\mathrm{P}_{\mathrm{i}}\right)^{1-3}$. When operating in reverse direction, $\mathrm{F}_{1}$ hydrolyzes ATP to rotate the rotor $\gamma$-subunit against the hexameric ring-shaped stator $\alpha_{3} \beta_{3}$. The sub-complex $\alpha_{3} \beta_{3} \gamma$ is the minimum component for $F_{1}$ to operate. $F_{1}$ has three catalytic sites located at the $\alpha \beta$ interfaces and $\varepsilon$ hosted mainly by the $\beta$-subunits. The rotation of the $\gamma$-subunit is induced by the transitions among catalytic states and cooperative conformation changes of the three $\beta$-subunits ${ }^{4,5}$. Precise torque experiments ${ }^{6-8}$ have shown that $F_{1}$ is a highly efficient motor that can convert almost all of the chemical free energy of hydrolysis to the mechanical energy of rotation.

To understand the working principles and the fine coordination of the conformation changes of the $\beta$-subunits, extensive studies have been carried out to unveil the tight chemomechanical couplings between the rotation and the catalytic states as shown in Fig. 1 for $\mathrm{F}_{1}$ derived from thermophilic Bacillus PS3. $\mathrm{F}_{1}$ performs counterclockwise step-like rotation (viewed from the membrane side) of $120^{\circ}$ steps each coupled with the hydrolysis of a single $\mathrm{ATP}^{6}$. The $120^{\circ}$ step further decomposes into $80^{\circ}$ and $40^{\circ}$ substeps $^{9}\left(85^{\circ}\right.$ and $35^{\circ}$ substeps for Escherichia coli $\left.\mathrm{F}_{1}-\mathrm{ATPase}^{10}\right)$. It was found that the $80^{\circ}$ substep is triggered by ATP binding and ADP release $9,11,12$ at different $\beta$-subunits, while the $40^{\circ}$ substep is induced by ATP hydrolysis and $\mathrm{P}_{\mathrm{i}}$-release ${ }^{12-14}$ that also occur at different $\beta$-subunits ${ }^{15,16}$ (see Fig. 1). The dwells before the $80^{\circ}$ and the $40^{\circ}$ substeps are termed the binding and the catalytic dwells, respectively.

On the other hand, single $F_{1}$ stall-and-release experiment ${ }^{17}$ shows that the catalytic rates of $F_{1}$ are modulated by the rotary angle of the $\gamma$-subunit. Specifically, the rate constants of ATP binding and $\mathrm{P}_{\mathrm{i}}$-release are found to be highly dependent on the $\gamma$-angle, suggesting that these two reactions are the major torquegenerating steps ${ }^{1,18}$. In contrast, the ATP hydrolysis is less affected by the $\gamma$ rotation and, therefore, does not contribute much to the torque-generation. Furthermore, quantum mechanical/molecular mechanical calculations ${ }^{19}$ demonstrate that the chemical free energy released from hydrolyzing a bound ATP at the $\mathrm{F}_{1}$ catalytic site is $\sim 10 \mathrm{pN} \cdot \mathrm{nm}$, which is relatively small compared with the free-energy $\Delta G$ released from hydrolyzing one ATP in a solution ${ }^{7,20}$, for example, $\Delta G \sim-90 \mathrm{pN} \cdot \mathrm{nm}$ under physiological conditions: [ATP] $\sim 1 \mathrm{mM}, \quad[$ ADP $] \sim 0.1 \mathrm{mM}$ and $\left[\mathrm{P}_{\mathrm{i}}\right] \sim 1 \mathrm{mM}$. With its insignificant contributions to the torque-generation and the overall chemical energy released, it remains elusive what functional role the hydrolysis of the bound ATP can play in the $\mathrm{F}_{1}$ catalytic cycle.

In this study, we investigate the detailed kinetics of the catalytic dwells and scrutinize the possible roles of the ATP hydrolysis reaction in terms of single $\mathrm{F}_{1}$ rotary observations with microsecond time resolutions and contemporary time series analysis. In particular, model-free change point (CP) and clustering analyses are applied to the angular traces from free rotations of wild-type (WT) $F_{1}$ derived from thermophilic Bacillus PS3 to robustly construct the statistics of waiting time and angular fluctuations of the catalytic dwells with short duration $\sim 1 \mathrm{~ms}$. This allows us to detect a small angular increment during the catalytic dwell triggered by the ATP hydrolysis reaction that is indiscernible in previous studies using conventional analysis methods. Moreover, we find in freely rotating $\mathrm{F}_{1}$ that ATP hydrolysis is followed by $\mathrm{P}_{\mathrm{i}}$-release with low synthesis rate compared with the hydrolysis rate. We then propose the functional roles of ATP hydrolysis as a key to accelerate (or kinetically unlock) the subsequent $\mathrm{P}_{\mathrm{i}}$-release reaction and promote the correct reaction ordering, despite its minor contributions to the torque and chemical energy generations.

\section{Results}

Observing the rotary motions of single $F_{1}$-ATPase. WT $F_{1}$ derived from thermophilic Bacillus PS3 are prepared as described in ref. 21. To monitor the rotary motions of $\mathrm{F}_{1}$, the stator complex $\left(\alpha_{3} \beta_{3}\right)$ is fixed to a glass surface and a colloidal gold bead as a rotation probe with diameters of $40-80 \mathrm{~nm}$ is attached to the rotor $\gamma$-subunit ${ }^{15}$. The freely rotating beads were then observed at room temperature under a custom-built laser dark-field microscope $e^{22}$. To focus on the catalytic dwells, the rotation assay was performed at high ATP concentration $([\mathrm{ATP}]=4 \mathrm{mM})$, such that the binding dwells become extremely short $(\sim 10 \mu \mathrm{s})$ and are negligible. With high image recording rates of 27,000-100,000 frames per s, typical rotary traces show clear pause and rotation regions. An example is shown in Fig. 2a.

Identifying catalytic dwells by $\mathrm{CP}$ and clustering analyses. To obtain the catalytic dwell statistics reliably from the rotary trace free from artefacts that may arise from common analysis methods (for example, thresholding and binning ${ }^{23-25}$, CP-detection

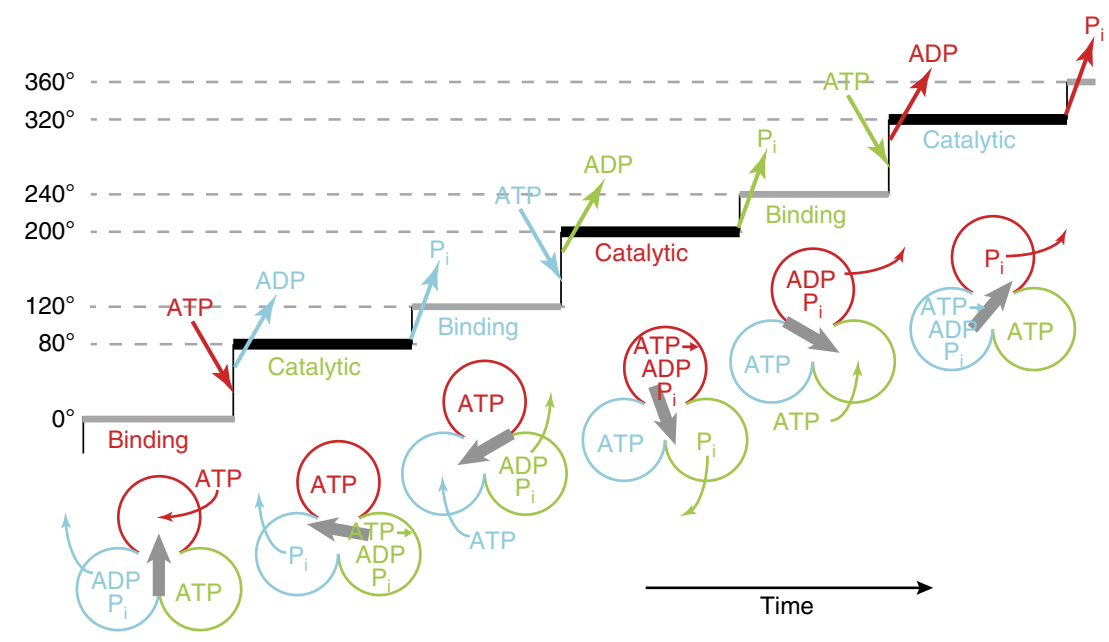

Figure 1 | Coupling between catalysis and rotation in $\mathbf{F}_{\mathbf{1}}$. Dwells in the $\gamma$ rotation and the corresponding nucleotide states at each $\beta$-subunit (coloured circles) are shown. Nucleotides are coloured according to the $\beta$-subunits for visual clarity. The grey arrows represent the rotary angles of the $\gamma$-subunit. ATP binding, hydrolysis, ADP release and $\mathrm{P}_{\mathrm{i}}$ release of the red $\beta$-subunit occur at $0^{\circ}, 200^{\circ}, 240^{\circ}$ and $320^{\circ}$, respectively. 
a
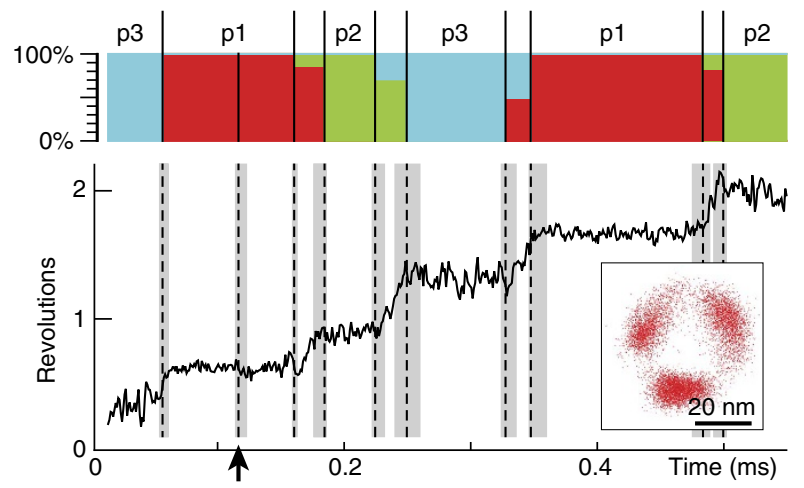

b
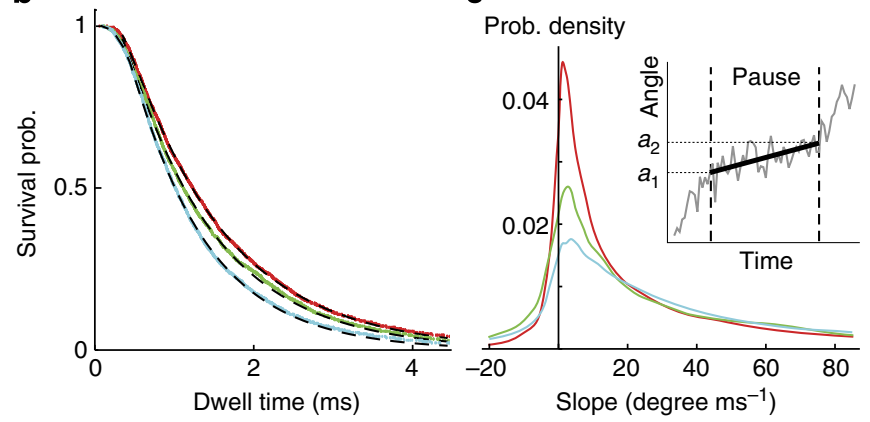

Notes 1-3, and validations in Supplementary Fig. 6). The uncertainties of $\mathrm{CP}$ locations are essential to estimate the corresponding errors in the catalytic dwell statistics. It is evident from Fig. 2a that the detection scheme can automatically identify CPs separating the pause and rotation segments in the time series.

Because of some undesired fluctuations in the measurements, extra CPs caused by actual bead motions may exist (for example, the $\mathrm{CP}$ indicated by the arrow in Fig. 2a). Therefore, $\mathrm{CP}$ detection is followed by a clustering procedure to classify the detected $\mathrm{CP}$ intervals into pause and rotation intervals, and to assign the pause intervals accordingly to the three different catalytic dwells separated by $120^{\circ}$. In this study, we employ an informationbased soft clustering method ${ }^{27,28}$ (detailed in Methods and Supplementary Note 4, and validations in Supplementary Figs 6,7) to assign each $\mathrm{CP}$ intervals to the three different catalytic dwells with the conditional probability, $P\left(C_{\alpha} \mid e_{i}\right)$, for a given CP interval $e_{i}\left(i=1, \ldots, N_{e}\right.$ with $N_{e}=$ total number of detected CP intervals) to belong to the catalytic dwell $C_{\alpha}(\alpha=1,2$ and 3). The clustering results are illustrated in the stacked bar chart in Fig. 2a with red, green and cyan bars labelling the conditional probabilities for a given $\mathrm{CP}$ interval to be assigned to the three catalytic dwells. Next, a CP interval is identified as a pause interval if it can be assigned to any catalytic dwell with $>95 \%$ probability, and is identified as rotation intervals otherwise. This corresponds to a possible 5\% assignment error for the pause intervals. Finally, extra CPs between consecutive pause intervals (for example, indicated by the arrow in Fig. 2a) assigned to the same catalytic dwell are removed.

Dwell-time statistics. The $\mathrm{CP}$ detection and clustering procedure results in a set of catalytic dwells from which various statistics can be extracted. Figure $2 \mathrm{~b}$ shows the dwell-time survival probabilities for each of the three catalytic dwells from a single $F_{1}$ attached to a $40 \mathrm{~nm}$ bead. In contrast to dwell-time histograms commonly used, survival probabilities are constructed in our analysis, since they do not require to introduce artificial binnings. The survival probabilities are well fitted by double exponential curves $\left(c_{1} \exp \left(-t / \tau_{1}\right)+c_{2} \exp \left(-t / \tau_{2}\right)\right)$ with two distinct time constants $\tau_{1} \sim 0.2 \mathrm{~ms}$ and $\tau_{2} \sim 1 \mathrm{~ms}$, which agrees with previous studies ${ }^{13}$ that there exist two catalytic processes (ATP hydrolysis and $\mathrm{P}_{\mathrm{i}}$-release) at the catalytic dwells. As can be seen from Fig. 2b, the observed time constants from different catalytic dwells of the same molecule vary, which can be caused by the heterogeneity of local environment around the motor. Therefore, dwell-times from different catalytic dwells are not mixed together in our analysis to avoid introducing spurious results. In addition, correlations between dwell times of successive catalytic pauses are investigated in terms of two-dimensional (2D) correlograms (Supplementary Fig. 9) and no apparent correlation can be detected.

To take into account the two observed time constants and the lack of correlations, we consider the four simplest scenarios for the catalytic dwell as shown in Fig. $2 \mathrm{~d}-\mathrm{g}$, where the chemical states of the catalytic dwell at $80^{\circ}$ in Fig. 1 is depicted as illustration. Specifically, the $\mathrm{P}_{\mathrm{i}}$-release (at cyan $\beta$-subunit) follows the hydrolysis (at green $\beta$-subunit) in Fig. $2 \mathrm{~d}-\mathrm{e}$, and vice versa in Fig. 2f-g. These imply that the power stroke connecting the current dwell with the next is triggered by the $\mathrm{P}_{\mathrm{i}}$-release in Fig. 2d,e and by the hydrolysis in Fig. 2f,g, respectively. Moreover, the $\mathrm{P}_{\mathrm{i}}$-release is rate-limiting in Fig. 2d,g, and the hydrolysis/ synthesis are rate-limiting in Fig. 2e,f. The rate constants, denoted by $k_{\text {hyd }}(\theta), k_{\text {syn }}(\theta)$ and $k_{\mathrm{Pi}}(\theta)$ for hydrolysis, synthesis and $\mathrm{P}_{\mathrm{i}}$-release, respectively, are in general $\gamma$-angle $(\theta)$ dependent ${ }^{17}$. In these schemes, the $P_{i}$ binding is ignored because of the low $P_{i}$ concentration considered in the current study. On the other 
hand, the synthesis reactions are neglected in Fig. 2f,g, where the power stroke is assumed to be triggered by the hydrolysis, implying that the chance of backward reaction (rotation) is low. In the following, we will show that our analyses of the angular fluctuations and their effects on the catalytic kinetics support the scenario in Fig. 2e with low synthesis rates.

Small rotation at the catalytic dwell. We first look at the slope distribution of the fitted linear trend of the pause intervals (Fig. 2c). The significant bias of distributions to the positive slopes indicates that on average there exists a small angular increment during the catalytic dwell. We propose that this angular increment originates from a small angular shift in the equilibrium angles (or free-energy minima) of the two catalytic states (for example, from the pre- to post-hydrolysis states in Fig. 2e) at the catalytic dwell. Although this angular increment is evident from the positive skewness of slope distribution, it is, however, too small to be resolved from angular histograms of the catalytic dwells considered in previous studies (Supplementary Fig. 8). We also note that the application of $\mathrm{CP}$ and clustering analyses to separate the rotation from the pause intervals is crucial in obtaining the correct slope distribution of the catalytic dwells free from false contribution from the rotation parts of the trace (see validations in Supplementary Figs 6,7). On the other hand, an angular shift after hydrolysis or $\mathrm{P}_{\mathrm{i}}$-release is not uncommon in $F_{1}$ motors. It was reported recently in human mitochondrial $\mathrm{F}_{1}$ (ref. 29) that the hydrolysis and $\mathrm{P}_{\mathrm{i}}$-release occur at separated dwells and trigger $\gamma$ rotations of $\sim 30^{\circ}$ and $\sim 25^{\circ}$, respectively.

Modelling the kinetics of the catalytic dwell. To integrate two catalytic states, the rate constants modulated by the $\gamma$-angle and the small angular increment at the catalytic dwell, we adopt a reaction-diffusion framework ${ }^{18,30}$ originally proposed in the study of electron transfer reaction under the effects of solvent orientation motions ${ }^{31}$. In this framework, the $\mathrm{F}_{1}$ catalytic kinetics is modelled by $2 \mathrm{D}$ reaction diagrams to account for the chemomechanical couplings between the catalytic reactions and rotary fluctuations. As a schematic illustration, the model is shown in Fig. 3a,b for the scheme in Fig. 2e with low synthesis rates (relative to hydrolysis). For each reaction (hydrolysis and $\mathrm{P}_{\mathrm{i}}$-release), the reaction diagram is characterized by two coordinates: the mechanical coordinate $(\gamma$-angle $\theta)$ and a 1D reactive coordinate ( $q_{\text {hyd }}$ and $q_{\mathrm{Pi}}$ ). The two reaction diagrams for hydrolysis and $\mathrm{P}_{\mathrm{i}}$-release are depicted separately since $q_{\mathrm{hyd}}$ and $q_{\mathrm{Pi}}$ are generally different. The free-energy surfaces of the catalytic states (the pre- and post-hydrolysis states), which are assumed to be harmonic in $\theta$, are represented by the equi-energy contours and intersect at the transition states $\left(\mathrm{TS}_{\mathrm{hyd}}\right.$ and $\left.\mathrm{TS}_{\mathrm{Pi}}\right)$ separating the reactant and product. The relaxations of $q_{\mathrm{hyd}}$ and $q_{\mathrm{Pi}}$ are assumed to be fast enough compared with the timescales of reactions and rotary fluctuations such that equilibrium in the reactive coordinate is always attained on the reactant's and product's surfaces.

A natural consequence from the inclusion of the mechanical coordinate $\theta$ in the reaction diagrams is that the activation energy depends on the $\gamma$-angle, giving rise to the angle-dependent rate constants $^{31}$. Moreover, we introduce an angular difference in the free energy minimum of the pre- and post-hydrolysis states (located at $\theta=-d_{1}$ and $d_{2}$ in Fig. 3a,b) to account for the small angular increment during the catalytic dwell. In the example of Fig. 3a,b, the $\gamma$-angle diffuses under the influence of the freeenergy surface in the reactant that describes the rotary potential exerted by the $\alpha_{3} \beta_{3}$ rings to the $\gamma$-subunit. During the diffusion along $\theta$, the system can react from the reactant to the product state with rate constants $k_{\mathrm{hyd}}(\theta)$ and $k_{\mathrm{Pi}}(\theta)$. We perform simulations of the reaction-diffusion dynamics of $\gamma$-angle on the free-energy surfaces in terms of the overdamped Langevin dynamics and Monte Carlo techniques, where the model parameters of reactions $\left(k_{\mathrm{hyd}}(\theta), \quad k_{\text {syn }}(\theta)\right.$ and $\left.k_{\mathrm{Pi}}(\theta)\right)$ and diffusion (relaxation time, angular increment and potential width) are extracted from the experimental data (detailed in Methods).

Following previous studies ${ }^{17}$, we adopt an exponential angle dependence for the rate constants of hydrolysis and $\mathrm{P}_{\mathrm{i}}$-release, $k_{\text {hyd }}(\theta) \propto \exp \left(b_{\text {hyd }} \theta\right)$ and $k_{\mathrm{Pi}}(\theta) \propto \exp \left(b_{\mathrm{Pi}} \theta\right)$ with $b_{\text {hyd }} \sim 0.02$ degree ${ }^{-1}$ and $b_{\mathrm{Pi}} \sim 0.12$ degree $^{-1}$, and an angular independent $k_{\text {syn }}(\theta)=k_{\text {syn. }}$. We note that $k_{\mathrm{Pi}}(\theta)$ has the strongest $\theta$ dependence (increases more than 10 times when $\theta$ increases $20^{\circ}$ ), implying that the $\mathrm{P}_{\mathrm{i}}$-release reaction is significantly decelerated when the viscous drag of the probe increases (that is, when the relaxation time of rotary fluctuations increases) as demonstrated recently by Watanabe et al. ${ }^{18}$. The hydrolysis and synthesis reactions, on the other hand, are insensitive to the viscous drag of the probe because of the weak $\theta$ dependence of $k_{\text {hyd }}(\theta)$ and $k_{\text {syn }}$.

The reaction diagrams for the other schemes in Fig. $2 \mathrm{~d}-\mathrm{g}$ are similar to the one in Fig. 3a,b. For example, for Scheme 4 of Fig. 2g, the reactant and product states in Fig. $3 \mathrm{~b}$ are replaced with the pre- and post- $\mathrm{P}_{\mathrm{i}}$-release states with the corresponding reactive coordinates and TSs swapped between Fig. 3a,b.

A schematic representation of the reaction-diffusion processes and the corresponding nucleotide states at the catalytic dwell is shown in Fig. 3a-c. Starting at step 1, the system diffuses in the pre-hydrolysis state during $\mathbf{1} \rightarrow \mathbf{2}$. Upon ATP hydrolysis $(\mathbf{2} \rightarrow \mathbf{3})$ at the green $\beta$-subunit in Fig. $3 c$, the system reacts to the posthydrolysis state, where the system diffuses during $\mathbf{3} \rightarrow \mathbf{4}$. Steps $\mathbf{1 - 4}$ correspond to the catalytic pause interval identified by $\mathrm{CP}$ detection for the experimental rotary traces, and a small angular increment $\left(\Delta \theta=d_{2}+d_{1}\right)$ is introduced upon the reaction $\mathbf{2} \rightarrow \mathbf{3}$. The current dwell ends at step $\mathbf{4}$ when $\mathrm{P}_{\mathrm{i}}$-release occurs $(\mathbf{4} \rightarrow \mathbf{5})$ to bring the system to the pre-hydrolysis state of the next catalytic dwell (near $120^{\circ}$ ). After landing at step 5 locating at the highenergy region of the potential, a power stroke $(5 \rightarrow 1)$ takes place and the system relaxes to around the potential minimum. The next catalytic dwell starts again at step $\mathbf{1}$ and the above process repeats.

Determining the rate-limiting step at the catalytic dwell. Rotary traces mimicking the $40 \mathrm{~nm}$ bead case are simulated for different schemes in Fig. $2 \mathrm{~d}-\mathrm{g}$. Typical traces are shown in Fig. $3 \mathrm{~d}-\mathrm{g}$ and the equilibrium angles of the states where the system resides are indicated by red lines. It is evident from the traces of the equilibrium angle that the nature of angular fluctuations at the catalytic dwells varies with the schemes. As there are two distinct time constants $(\sim 0.2$ and $1 \mathrm{~ms})$ obtained by experiments at the catalytic dwell for the case of $40 \mathrm{~nm}$ bead, there are three distinct types of angular fluctuations: (1) the equilibrium angle frequently switches between the minima at $\theta=-d_{1}$ and $d_{2}$ during the catalytic dwell (Fig. 3d). This corresponds to Scheme 1 where the system can make frequent transitions between the pre- and posthydrolysis states because of slow $\mathrm{P}_{\mathrm{i}}$-release; (2) the equilibrium angle stays long at $\theta=-d_{1}$ and then briefly at $\theta=d_{2}$ (Fig. 3e,g). This corresponds to the cases where the reverse reaction rarely occurs and the first process at the catalytic dwell is rate-limiting; and (3) the equilibrium angle stays shortly at $\theta=-d_{1}$ but for a longer time at $\theta=d_{2}$ (Fig. 3f). This corresponds to the cases where the reverse reaction rarely occurs and the second process at the catalytic dwell is rate-limiting.

Compared with the experimental data, these differences in the angular fluctuations provide useful clue in identifying the correct 
a

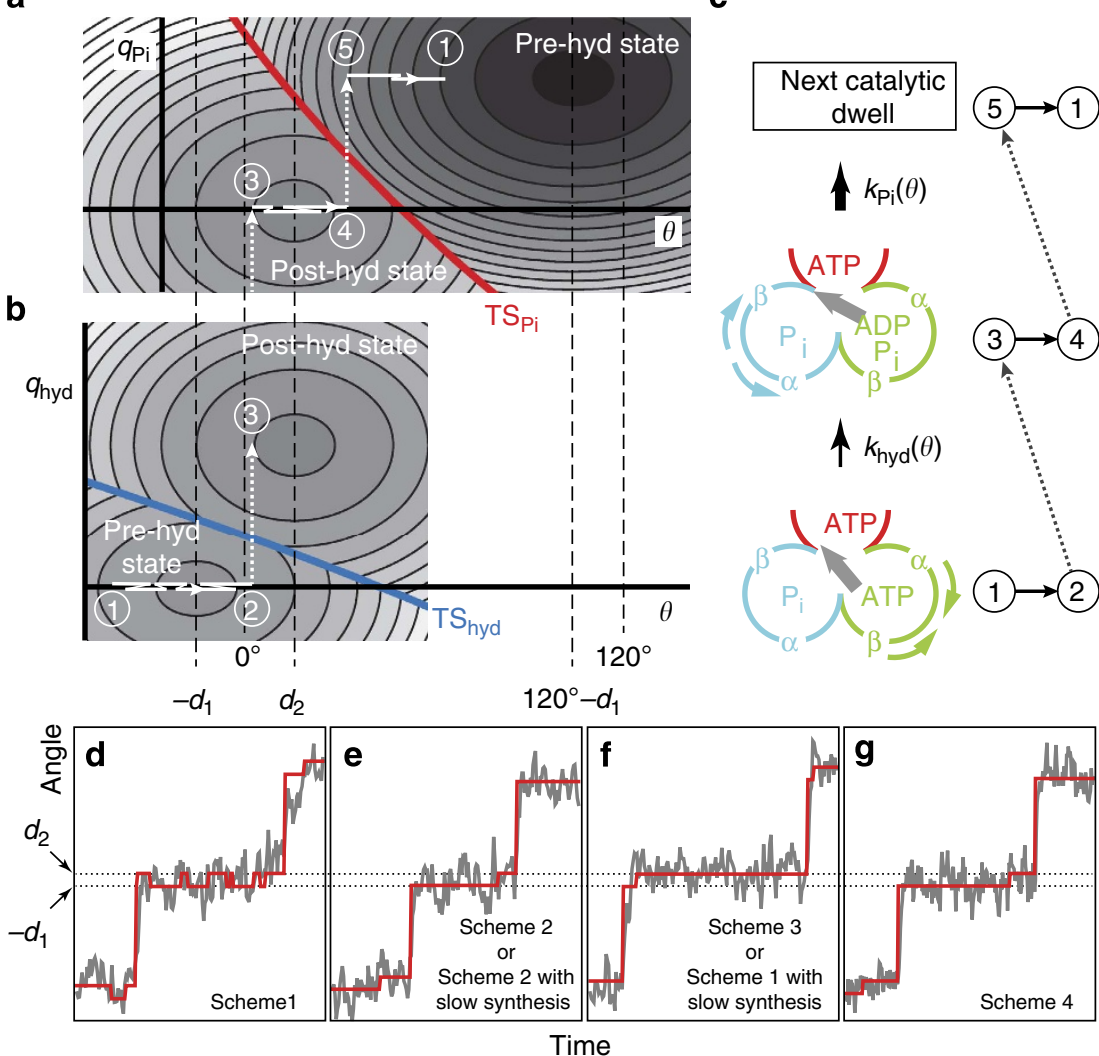

Figure 3 | Free-energy surfaces for the catalytic dwell and typical simulation traces. (a-b) 2D $P_{i}$-release (a) and hydrolysis (b) reaction diagrams for the scheme in Fig. $2 \mathrm{e}$ with slow synthesis (not to scale). $q_{\mathrm{hyd}}$ and $q_{\mathrm{Pi}}$ are reactive coordinates. The post-hydrolysis state on the product side for the hydrolysis is redrawn on the reactant side for the $\mathrm{P}_{\mathrm{i}}$-release. Free-energy surfaces are represented by equi-energy contours with darker colours for lower energies. The reactant and product surfaces intersect at $\mathrm{TS}_{\text {hyd }}$ and $\mathrm{TS}_{\mathrm{Pi}}$. The catalytic dwells locate around $\theta=0^{\circ}$ and $120^{\circ}$, and the equilibrium angles of the pre- and post-hydrolysis states locate at $\theta=-d_{1}$ and $d_{2}$, respectively. A schematic representation of reaction-diffusion path during one catalytic dwell is shown with numberings by white solid (diffusion) and dash (reaction) lines $\left(\mathbf{1} \rightarrow \mathbf{2}\right.$ and $\mathbf{3} \rightarrow \mathbf{4}$ : diffusion along $\theta ; \mathbf{2} \rightarrow \mathbf{3}$ and $\mathbf{4} \rightarrow \mathbf{5}$ : hydrolysis and $\mathrm{P}_{\mathrm{i}}$-release reaction; $\mathbf{5} \rightarrow \mathbf{1}$ : power stroke). For visual clarity, small offsets are introduced in the white solid lines for the diffusion along $\theta$. (c) Correspondence between the chemical states and the numbered trajectory in $\mathbf{a}, \mathbf{b}$. The relative orderings of the $\alpha$ - and $\beta$-subunits and the associated conformational changes at the catalytic dwell (see Discussion for details) are also indicated. (d-g) Typical simulated traces (for $40 \mathrm{~nm}$ bead case) of different schemes (with synthesis rates comparable to or much lower than the hydrolysis rates) in Fig. $2 \mathrm{~d}-\mathrm{g}$. Subtitles indicate the corresponding scheme(s) for the traces. Grey lines: simulated rotary trace. Red lines: equilibrium angle of the states where the system resides.

scheme for the catalytic dwell. In particular, we consider the statistics of initial- and final-angle of the linear trend of the pause intervals ( $a_{1}$ and $a_{2}$ defined in Fig. 2c) to differentiate the above three types of angular fluctuations. The initial- and final-angle distributions for the four simulation cases in Fig. 3d-g are shown, respectively, in Fig. 4a-d, and they can be distinguished by the widths of their initial- and final-angle distributions. To quantify the degree of statistical dispersion, we evaluate the median absolute deviation (MAD) that is less influenced by outliers and skewness of the distribution ${ }^{32}$. For a list of values $\left\{x_{1}, x_{2}, \cdots, x_{n}\right\}$ with median $\tilde{x}, \mathrm{MAD}$ is the median of the list $\left\{\left|x_{1}-\tilde{x}\right|,\left|x_{2}-\tilde{x}\right|, \cdots,\left|x_{n}-\tilde{x}\right|\right\}$. In Fig. 4f, we compare the ratios of final- and initial-angle MADs between the experimental data with $40 \mathrm{~nm}$ bead and those from simulations. It is clear that the experimental data favours the initial- and final-distributions given in Fig. $4 \mathrm{~b}, \mathrm{~d}$, that is, the second type of angular fluctuations in Fig. 3e,g corresponding to Scheme 2, Scheme 2 with slow synthesis and Scheme 4 . This result suggests that the rate-limiting step is the first catalytic process at the catalytic dwell. Moreover, in order for the traces in Fig. 3e,g to produce a ratio of final- and initial-angle MADs matching the experimental ones $(\sim 1.2)$, an angular increment $\Delta \theta=d_{2}+d_{1} \sim 20^{\circ}$ is required (see Methods).
It remains to determine which process (hydrolysis or $\mathrm{P}_{\mathrm{i}}$-release) proceeds first at the catalytic dwell.

Hydrolysis followed by $\mathbf{P}_{\mathrm{i}}$-release with low synthesis rates. Since the angular fluctuations in Fig. 3e,g are quite similar for the case of $40 \mathrm{~nm}$ bead, other features of the reaction-diffusion systems are needed to determine the ordering of hydrolysis and $\mathrm{P}_{\mathrm{i}}$-release. Here we use the strong sensitivity of $\mathrm{P}_{\mathrm{i}}$-release reaction to the relaxation time of angular fluctuations for the identification. By performing single $F_{1}$ rotary measurements with various bead sizes $(40,60$ and $80 \mathrm{~nm})$ under the same experimental conditions, we monitor the changes of the two observed time constants at the catalytic dwell as a function of relaxation time as shown in Fig. 4g,h, where the relaxation times of the experimental rotary traces are determined by evaluating the autocorrelation of angular fluctuation at the catalytic dwells. Figure $4 \mathrm{~g}-\mathrm{h}$ show that the smaller time constant from the single $\mathrm{F}_{1}$ data is sensitive to the relaxation time, whereas the larger one does not. In the same figure, we also show the dependence of time constants on the relaxation time from simulations. Only Scheme 2 with slow synthesis compared with the hydrolysis can explain the strong 
a

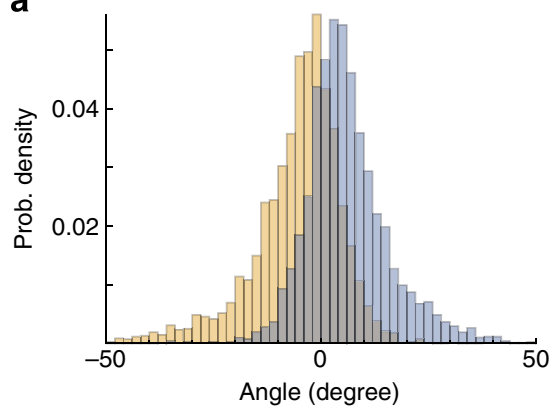

C

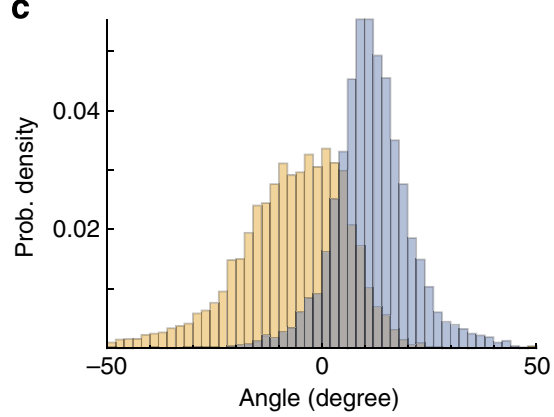

e

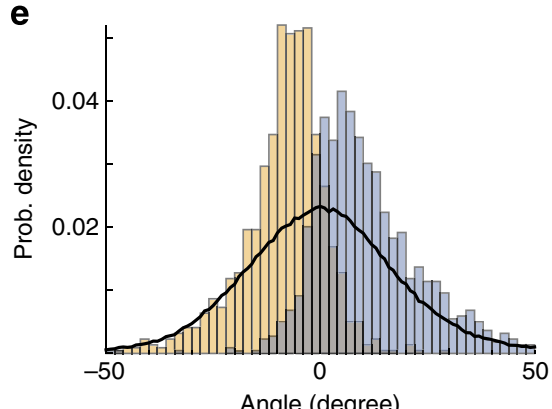

g

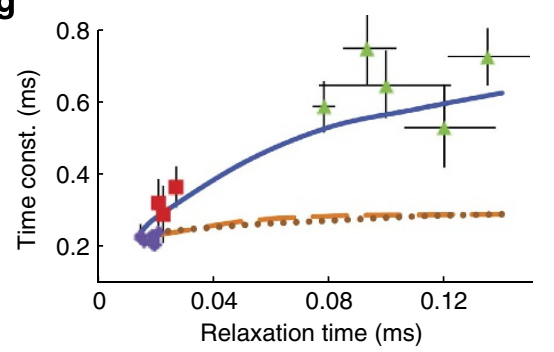

b

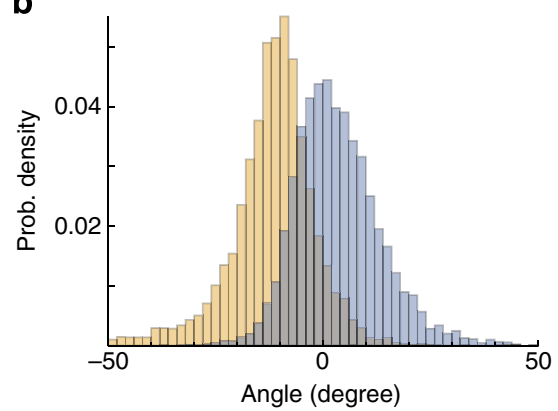

d

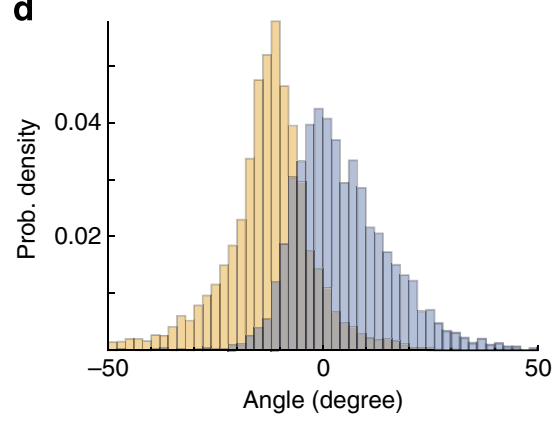

f

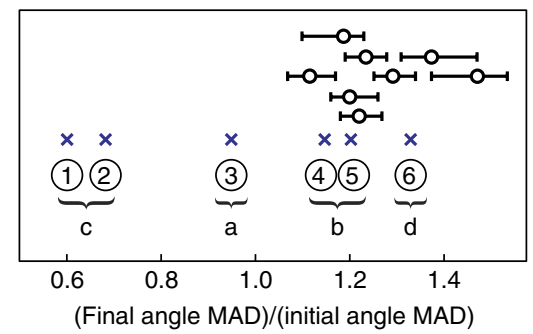

h

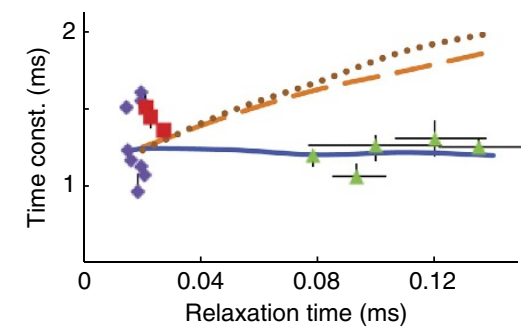

\begin{tabular}{|c|c|c|c|}
\hline & Single F1 data: $40 \mathrm{~nm}$, & $60 \mathrm{~nm}, \quad \Delta 8$ & $\mathrm{~nm}$ \\
\hline Simulation: & - Scheme 2, & $\begin{array}{l}\text { Scheme } 2 \text { with } \\
\text { slow synthesis, }\end{array}$ & ...... Scheme 4 \\
\hline
\end{tabular}

Figure 4 | Initial and final angle distributions and relaxation time dependence of catalytic time constants. (a-d) Initial- (yellow) and final-angle (grey) distributions for the four simulation cases (with $40 \mathrm{~nm}$ bead) in Fig. 3d-g. (e) Typical initial- and final-angle distributions from one catalytic dwell of a single $\mathrm{F}_{1}$ (with $40 \mathrm{~nm}$ bead). Solid line: angle distribution of the catalytic dwell. (f) Ratios of the final- and initial-angle MAD from experimental data with $40 \mathrm{~nm}$ bead (circles) and simulations (crosses). Numbering of crosses: (1) Scheme 3; (2) Scheme 1 with slow synthesis; (3) Scheme 1; (4) Scheme 2;

(5) Scheme 2 with slow synthesis; and (6) Scheme 4. The corresponding initial- and final-angle distributions are also indicated. Error bars represent the $68 \%$ confidence intervals of the MAD ratio. ( $\mathbf{g}, \mathbf{h}$ ) Catalytic time constants versus relaxation time of angular fluctuations from experiments and simulations. Smaller time constant $(\mathbf{g})$ and for the Larger one $(\mathbf{h})$. Error bars: uncertainties in the estimated relaxation times and time constants fitted from rotary traces. Each point in $\mathbf{f}-\mathbf{h}$ corresponds to one catalytic dwell from a single $F_{1}$.

sensitivity of the smaller time constant on the relaxation time, since in that case the smaller time constant corresponds to the faster $\mathrm{P}_{\mathrm{i}}$-release reaction (Fig. 2e). In contrast, the smaller time constant from Scheme 4 corresponds to the hydrolysis reaction (Fig. 2g), which is insensitive to the relaxation time.

The situation is more subtle in the case of Scheme 2. Since Scheme 2 contains the synthesis (backward) reaction (Fig. 2e), the smaller time constant roughly characterizes reaction paths that do not involve the backward reaction, that is, time constant for hydrolysis directly followed by $\mathrm{P}_{\mathrm{i}}$-release, and the larger time constant corresponds to reaction paths that involve several hydrolysis and synthesis reactions (Fig. 2e). As the relaxation time of angular fluctuation increases, the $\mathrm{P}_{\mathrm{i}}$-release reaction decelerates significantly which causes an increase in the number of cycles of 
hydrolysis and synthesis reactions, resulting in the strong sensitivity of the larger time constant on the relaxation time.

Our result, that $\mathrm{P}_{\mathrm{i}}$-release is the process just before the power stroke, is consistent with the expectation ${ }^{7,15}$ that $\mathrm{P}_{\mathrm{i}}$-release, instead of the hydrolysis, is the major torque-generation step at the catalytic dwell attributed to the strong angular dependence of $k_{\mathrm{Pi}}(\theta)$.

Role of hydrolysis as a key to accelerate $\mathbf{P}_{\mathbf{i}}$-release. Figure $3 \mathrm{a}-\mathrm{c}$ provide a summary of the above results in terms of the $2 \mathrm{D}$ reaction diagrams. With a strong angular dependence of $k_{\mathrm{Pi}}(\theta)$, it can be seen from the figure that the angular increment from pre- to post-hydrolysis states brings the system towards the lower activation barrier regions of the $\mathrm{P}_{\mathrm{i}}$-release $\mathrm{TS}_{\mathrm{Pi}}$ to react. The angular increment, $\Delta \theta=d_{1}+d_{2} \sim 20^{\circ}$, accelerates the $\mathrm{P}_{\mathrm{i}}$-release reaction more than 10 times as if the system would cross the $\mathrm{P}_{\mathrm{i}}$-release activation barrier at the pre-hydrolysis state, that is, at $\theta \sim-d_{1}$. We therefore propose a functional role of hydrolyzing the bound ATP as a 'key' to accelerate (or kinetically 'unlock') the subsequent $\mathrm{P}_{\mathrm{i}}$-release reaction to complete the catalytic cycle, even though the hydrolysis process is not a major torquegenerating step and the chemical free energy produced is notably small compared with those from the ATP binding and $\mathrm{P}_{\mathrm{i}}$-release reactions.

Another implication from the reaction diagrams in Fig. 3a,b and the role of hydrolysis as a key is the promotion of correct reaction ordering at the catalytic dwell, that is, the $\mathrm{P}_{\mathrm{i}}$-release (the torque-generating step) should follow the hydrolysis to avoid rotating away from the catalytic dwell before the hydrolysis of ATP occurs. More precisely, suppose the system is at the prehydrolysis state with $\theta \sim-d_{1}$ and it can proceed either with the hydrolysis (at the green $\beta$-subunit) or $\mathrm{P}_{\mathrm{i}}$-release (at the cyan $\beta$-subunit) by crossing the $\mathrm{TS}_{\mathrm{hyd}}$ or $\mathrm{TS}_{\mathrm{Pi}}$ (Fig. 3a-c). A simple estimation using the angle dependence of the rate constants (see Methods) shows that $k_{\text {hyd }}\left(\theta=-d_{1}\right)$ is more than 10 times larger than $k_{\mathrm{Pi}}\left(\theta=-d_{1}\right)$. This implies that it is unlikely to have the $\mathrm{P}_{\mathrm{i}}$-release and power stroke occurring before the hydrolysis (that is, turning the key).

\section{Discussion}

By extracting reliable dwell statistics from high-speed singlemolecule imagings in terms of contemporary $\mathrm{CP}$ and clustering analyses, the aims of the present study are to reveal the detailed kinetics and the possible roles of bound ATP hydrolysis at the catalytic dwells of WT $F_{1}$ free from any applied stalling force. To establish a connection with the conformational changes of the $\alpha$ - and $\beta$-subunits, we correlate our results with recent conformational studies of $\mathrm{F}_{1}$ in the following. It was found $\mathrm{d}^{16,33,34}$ that the principal conformation changes at the $\alpha_{3} \beta_{3}$ stator ring during the catalytic cycle involve the close/open transitions of the $\beta$-subunits upon nucleotide bindings/release, and the tighten/loosen transitions of the $\alpha \beta$ interfaces upon hydrolysis and $\mathrm{P}_{\mathrm{i}}$-release. In particular, structural comparison of $\mathrm{F}_{1}$ crystal structures $^{16}$ and molecular dynamics simulation ${ }^{34}$ show that at the catalytic dwell shown in Fig. 3c, the $\alpha \beta$ interface (green $\alpha$ and $\beta$ ) with ATP bound at $200^{\circ}$ before becomes tighter to facilitate hydrolysis. We expect that this prominent tightening motion can only induce a small $\gamma$-angle increment $\left(\sim 20^{\circ}\right.$ suggested by our analysis) from pre- to post-hydrolysis states (Fig. 3c), probably due to the weak interaction between the $\beta$ - and $\gamma$-subunits during the hydrolysis reaction, as implied by the weak $\gamma$-angle dependence of $k_{\text {hyd }}(\theta)$ (ref. 17) and the recent finding ${ }^{35}$ that the major contact region at the $\beta$-subunit (a conserved DELSEED helical loop located at the C-terminal domain of the $\beta$-subunit) with the $\gamma$-subunit does not contribute much to the torque transmission associated with the ATP hydrolysis reaction.

On the other hand, structural and conformational studies ${ }^{36-38}$ also suggest that at the catalytic dwell the $\beta$-subunit which bound ATP at $200^{\circ}$ before (green $\beta$ in Fig. $3 c$ ) strongly interacts with the two neighbouring $\alpha$-subunits (green and cyan $\alpha$ 's in Fig. $3 c$ ) such that motions of the two $\alpha \beta$ interfaces (green and cyan) well correlate with each other. In particular, the tightening of the green $\alpha \beta$ interface for hydrolysis is expected to trigger the loosening of the cyan $\alpha \beta$ interface in Fig. 3c, resulting in a decrease in the binding affinity of $\mathrm{P}_{\mathrm{i}}$ (ref. 16) to be released at the end of the catalytic dwell. We therefore propose that the role of bound ATP hydrolysis as a key to assist the release of $P_{i}$ may correspond to this tightly correlated $\alpha \beta$ interface motions. Similarly, without the occurrence of the hydrolysis reaction, the $\alpha \beta$ interface with the bound $P_{i}$ (cyan $\alpha \beta$ in Fig. 3c) would remain tight and thus, it is unlikely for the $\mathrm{P}_{\mathrm{i}}$-release to happen. Furthermore, the close interplay between couplings among different subunits in the $\alpha_{3} \beta_{3}$ stator rings and the fine coordination of reaction sequence does not occur only at the catalytic dwell. It was observed using high-speed atomic force microscopy ${ }^{38,39}$ that the $\alpha_{3} \beta_{3}$ stator ring still undergoes cyclic conformation changes even without the rotor $\gamma$-subunit, in which the open-to-close transition of the $\beta$-subunit induced by the ATP binding also behaves like a key to expedite the close-to-open transition of an adjacent $\beta$-subunit for the release of ADP. We therefore expect that such unlock mechanism could be a common strategy to achieve precise chemomechanical coordination in the $F_{1}$. It was reported ${ }^{29}$, however, in human mitochondrial $F_{1}$ that $\mathrm{P}_{\mathrm{i}}$-release (at cyan $\beta$ in Fig. 3c) precedes hydrolysis (at green $\beta$ in Fig. 3c). In that case, the release of $P_{i}$ may instead serve as the key to expedite the ATP hydrolysis.

Finally, we point out that the slow synthesis reaction at the catalytic dwell suggested here from free rotation is different from previous single $\mathrm{F}_{1}$ observations with applied force to stall the rotation of the $\gamma$-subunit ${ }^{15,17}$ for a long enough time, where the rate constant of synthesis are found to be comparable with those of the hydrolysis. We speculate that this difference may originate from some non-equilibrium features of the freely rotating WT $F_{1}$ at the short $(\sim \mathrm{ms})$ catalytic dwell. A precise estimation of the synthesis rates under non-equilibrium conditions will be focused in the future studies.

\section{Methods}

Data-selection criteria. Rotary traces from measurements are first plotted on the camera $x-y$ plane to check for circular symmetry. Traces significantly deviated from a circle are not used in the analysis, since the system may be affected by unknown factors such as interaction of the probe with the glass surface, improper fixation, defected molecule and so on. After the above selection, five molecules from the $40 \mathrm{~nm}$ bead case, four molecules from the $60 \mathrm{~nm}$ case and five molecules from the $80 \mathrm{~nm}$ case are considered. The number of pause intervals associated with a single catalytic dwell from any single $F_{1}$ molecule is in general larger than 1,500. On the other hand, catalytic dwells having extremely distinct statistics, that is, outliers, from the majority are not included in analysis.

Curve fitting. The least squares fitting is employed in our study to fit survival probabilities and autocorrelation functions with exponential functions. The number of exponentials is varied starting from one. The appropriate number of exponentials is then determined as the squared error between the fitted function and the survival probabilities (or autocorrelation function) becomes unchanged as the number of exponentials increases.

Change point analysis. CP analysis based on permutation test ${ }^{26}$ is generalized to detect changes of linear trend in the rotary traces. A CP is the time instant where the linear trends are different before and after. The major steps in CP detection are as follows.

Firstly, testing the existence of CPs by permutation method. CP detection is treated as a statistical hypothesis test with the two hypotheses: no CP exists versus at least one CP exists. In terms of permutation method, the likelihood that CPs 
exist in the trace is compared with the likelihood that there is no CP. We declare the existence of CPs if the hypothesis of no $\mathrm{CP}$ can be rejected when a given confidence level, for example, $98 \%$, is reached. The method to test the existence of CPs is discussed in detail in the Supplementary Note 1 and Supplementary Figs 1,2.

Secondly, determining the location of change point and its uncertainty. Once the existence of a $\mathrm{CP}$ is confirmed, the most probable location of the $\mathrm{CP}$ is assigned as the time instant whose left and right segments are best fitted by linear trends in terms of the squared errors. Moreover, the uncertainty in CP location is estimated using the bootstrapping method ${ }^{40}$. The size of the uncertainty depends on both the signal-to-noise ratio of the time series and the number of data points before and after the CP. Since uncertainties in the location of the detected CPs directly affect the dwell-time statistics, we keep track of the error by propagating ${ }^{41}$ the error in the CP location to the dwell time, that is, the time difference between two successive CPs. The set of dwell times and their uncertainties are then subjected to the subsequent fitting to determine the time constants and their uncertainties. The method to determine the CP location and its uncertainty is discussed in detail in the Supplementary Note 2 and Supplementary Fig. 3.

Thirdly, detecting multiple change points recursively. The above steps are repeated with binary segmentation to detect multiple CPs in the rotary trace. The segmentation stops when no new $\mathrm{CP}$ can be found with the given confidence level. The method to detect multiple CPs is discussed in detail in the Supplementary Note 3 and Supplementary Fig. 4.

Clustering to assign CP intervals to catalytic dwells. Here we employ a soft clustering algorithm based on the rate distortion theory in information theory ${ }^{27,28}$. The basic idea behind the algorithm is to treat clustering as 'compressing' the description of data set (the set of CP intervals in our case) into clusters (the three catalytic dwells in our case), while maintaining the distortion to a desired level. Owing to the fact that different catalytic dwells have separated distributions of the rotary angle, the distortion is chosen in our analysis to be the average intra-cluster angle difference in the resulting clusters. Generally, if one compresses the data set, for example, by using a few number of clusters, some details of the data set may be lost, implying the compressed description is 'distorted' compared with the data set. Therefore, it is always a tug-of-war between compression and maintaining small distortion. A self-contained discussions of the theory, properties and scheme to perform soft clustering of the $\mathrm{CP}$ intervals into different catalytic dwells are detailed in the Supplementary Note 4 and Supplementary Fig. 5. The validations of $\mathrm{CP}$ and clustering analyses on simulated traces are given in Supplementary Figs 6,7.

Numerical simulation of the reaction-diffusion model. We simulate the diffusion of the $\gamma$-angle $\theta$ at a reaction state (for example, the pre-hydrolysis state) on the $2 \mathrm{D}$ reaction diagram by the overdamped Langevin equation on a harmonic well, $d \theta / d t=-\lambda\left(\theta-\theta_{\mathrm{e}}\right)+R(t)$. Here $\theta_{\mathrm{e}}$ is the equilibrium angle, $\lambda=\omega^{2} / \eta$ $(\eta$ : friction coefficient, $\omega$ : frequency of the harmonic well) is the drift coefficient characterizing the relaxation time of $\theta$ (that is, $\langle\theta(t) \theta(0)\rangle \sim \exp (-\lambda t))$. $R(t)$ is a Gaussian random force exerting on $\theta$ with mean $\langle R(t)\rangle=0$ and correlation $\left\langle R(t) R\left(t^{\prime}\right)\right\rangle=2 \lambda \sigma^{2} \delta\left(t-t^{\prime}\right)$, where $\sigma^{2}$ is the variance of the equilibrium angular distribution (that is, normal distribution) at the harmonic well that is assumed to be the same for all catalytic states. The equilibrium angles of the two catalytic states are set to $n \times 120^{\circ}-d_{1}$ and $n \times 120^{\circ}+d_{2}$ with integer $n$ (see Fig. 3a,b in main text). For simplicity, we set $\left|d_{1}\right|=\left|d_{2}\right|=d$ in the simulation. We estimate the angular increment of the equilibrium angle, $\Delta \theta=d_{2}+d_{1}=2 d$, to be $\sim 20^{\circ}$ by requiring the ratios of final- and initial-angle MADs obtained from simulating the cases with sharper initial-angle distribution (that is, Scheme 2, Scheme 2 with slow synthesis and Scheme 4 in Fig. 2e,g) match the ratio obtained from experimental data (that is, $\sim 1.2$ shown in Fig. $4 \mathrm{f}$ ). Note that the ratios of the initial- and final-angle MADs from simulations significantly deviate from the ratios obtained from experiment for $\Delta \theta \lesssim 15^{\circ}$ or $\Delta \theta \gtrsim 25^{\circ}$.

Several values of $\lambda$ are used so that the relaxation timescale $\lambda^{-1}$ of the simulation covers the same range of relaxation timescales of $\theta$ from experiments, that is, $0.02 \mathrm{~ms} \lesssim \lambda^{-1} \lesssim 0.14 \mathrm{~ms}$ (see Fig. $4 \mathrm{~g}, \mathrm{~h}$ ). On the other hand, with $\Delta \theta$ chosen to be $20^{\circ}$, the variance $\sigma^{2}$ is set to 220 degree $^{2}$ such that the s.d. of the overall simulated angular distribution of the catalytic dwell $\left(\sim 17.5^{\circ}\right)$ match those from the experimental angular distributions. Note that only the pause intervals identified by the $\mathrm{CP}$ and clustering analyses are used to construct the experimental angular distributions in order to avoid possible artifacts from including the rotation intervals.

On the other hand, the reaction processes are simulated by Monte Carlo techniques as described before ${ }^{18}$. In particular, the angle dependence of the rate constants are adopted from previous experimental studies ${ }^{17}$ as $k_{\text {hyd }}=a_{\text {hyd }}$ $\exp \left(b_{\mathrm{hyd}} \theta\right), k_{\mathrm{Pi}}=a_{\mathrm{Pi}} \exp \left(b_{\mathrm{Pi}} \theta\right)$ and $k_{\text {syn }}=a_{\text {syn }}$, with $b_{\text {hyd }}=0.02$ degree $^{-1}, b_{\mathrm{Pi}}=0.12$ degree $^{-1}$ and $\theta=0^{\circ}$ corresponding to the angular position of the current catalytic dwell. We set $a_{\text {syn }}=a_{\text {hyd }}$ for the cases of Schemes 1 and 2 in Fig. $2 \mathrm{~d}$,e, and $a_{\text {syn }}=0$ for the cases of Schemes 1 and 2 with slow synthesis. The remaining constants $a_{\text {hyd }}$ and $a_{\mathrm{Pi}}$ are chosen such that the two time constants from the dwell-time distribution of the simulated traces reproduce those fitted from experimental dwell-time distributions with $40 \mathrm{~nm}$ bead, that is, purple points in Fig. 4g,h. The numerical values of $a_{\mathrm{hyd}}$ and $a_{\mathrm{Pi}}$ used in Fig. 4g,h are given as follows: (1) Scheme 2: $a_{\mathrm{hyd}}=1,200 \mathrm{~s}^{-1}=a_{\mathrm{syn}}$ and $a_{\mathrm{Pi}}=330 \mathrm{~s}^{-1}$; (2) Scheme 2 with slow synthesis: $a_{\mathrm{hyd}}=890 \mathrm{~s}^{-1}$ and $a_{\mathrm{Pi}}=330 \mathrm{~s}^{-1}$; and (3) Scheme $4: a_{\mathrm{hyd}}=3,600 \mathrm{~s}^{-1}$ and $a_{\mathrm{Pi}}=300 \mathrm{~s}^{-1}$.

In terms of Monte Carlo method, we simulate the transition of the system from one state to another according to the probability of making the corresponding state transition at each simulation step given by $P(\theta(t))=k(\theta(t)) \times \delta t$, where $\theta(t)$ is the $\gamma$-angle at the current simulation time step, $k(\theta(t))$ is the corresponding angledependent rate constant for hydrolysis, synthesis or $\mathrm{P}_{\mathrm{i}}$-release and $\delta t$ is the time interval between simulation steps. Moreover, we simulate long rotary trajectories that contain a large number of catalytic dwells ( $>10^{4}$ dwells) to ensure good sampling for the simulated dwell-time statistics.

\section{References}

1. Boyer, P. D. The ATP synthase-a splendid molecular machine. Annu. Rev. Biochem. 66, 717-749 (1997)

2. Yoshida, M., Muneyuki, E. \& Hisabori, T. ATP synthase-a marvelous rotary engine of the cell. Nat. Rev. Mol. Cell Biol. 2, 669-677 (2001).

3. Weber, J. Structural biology: toward the ATP synthase mechanism. Nat. Chem. Biol. 6, 794-795 (2010).

4. Wang, H. \& Oster, G. Energy transduction in the $F_{1}$ motor of ATP synthase. Nature 396, 279-282 (1998).

5. Mukherjee, S. \& Warshel, A. Electrostatic origin of the mechanochemical rotary mechanism and the catalytic dwell of $\mathrm{F}_{1}$-ATPase. Proc. Natl Acad. Sci. USA 108, 20550-20555 (2011).

6. Yasuda, R., Noji, H., Kinosita, K. J. \& Yoshida, M. $\mathrm{F}_{1}$-ATPase is a highly efficient molecular motor that rotates with discrete $120^{\circ}$ steps. Cell $\mathbf{9 3}$, 1117-1124 (1998).

7. Kinosita, K. J., Adachi, K. \& Itoh, H. Rotation of $F_{1}$-ATPase: how an ATP-driven molecular machine may work. Annu. Rev. Biophys. Biomol. Struct. 33, 245-268 (2004).

8. Toyabe, S. et al. Nonequilibrium energetics of a single $\mathrm{F}_{1}$-ATPase molecule. Phys. Rev. Lett. 104, 198103 (2010).

9. Yasuda, R., Noji, H., Yoshida, M., Kinosita, K. J. \& Itoh, H. Resolution of distinct rotational substeps by submillisecond kinetic analysis of $F_{1}$-ATPase Nature 410, 898-904 (2001).

10. Bilyard, T. et al. High-resolution single-molecule characterization of the enzymatic states in Escherichia coli $\mathrm{F}_{1}$-ATPase. Philos. Trans. R. Soc. Lond. B Biol. Sci. 368, 20120023 (2012).

11. Nishizaka, T. et al. Chemomechanical coupling in $\mathrm{F}_{1}$-ATPase revealed by simultaneous observation of nucleotide kinetics and rotation. Nat. Struct. Mol. Biol. 11, 142-148 (2004).

12. Adachi, K. et al. Coupling of rotation and catalysis in $\mathrm{F}_{1}$-ATPase revealed by single-molecule imaging and manipulation. Cell 130, 309-321 (2007).

13. Shimabukuro, K. et al. Catalysis and rotation of F1 motor: cleavage of ATP at the catalytic site occurs in $1 \mathrm{~ms}$ before $40^{\circ}$ substep rotation. Proc. Natl Acad. Sci. USA 100, 14731-14736 (2003).

14. Ariga, T., Muneyuki, E. \& Yoshida, M. $\mathrm{F}_{1}$-ATPase rotates by an asymmetric, sequential mechanism using all three catalytic subunits. Nat. Struct. Mol. Biol. 14, 841-846 (2007).

15. Watanabe, R., Iino, R. \& Noji, H. Phosphate release in $F_{1}$-ATPase catalytic cycle follows ADP release. Nat. Chem. Biol. 6, 814-820 (2010).

16. Okazaki, K. \& Takada, S. Structural comparison of $F_{1}$-ATPase: interplay among enzyme structures, catalysis, and rotations. Structure. 19, 588-598 (2011).

17. Watanabe, R. et al. Mechanical modulation of catalytic power on $\mathrm{F}_{1}$-ATPase. Nat. Chem. Biol. 8, 86-92 (2012).

18. Watanabe, R., Hayashi, K., Ueno, H. \& Noji, H. Catalysis-enhancement via rotary fluctuation of $\mathrm{F}_{1}$-ATPase. Biophys. J. 105, 2385-2391 (2013).

19. Hayashi, S. et al. Molecular mechanism of ATP hydrolysis in $F_{1}$-ATPase revealed by molecular simulations and single-molecule observations. J. Am. Chem. Soc. 134, 8447-8454 (2012).

20. Turina, P., Samoray, D. \& Gräber, P. $\mathrm{H}^{+} /$ATP ratio of proton transportcoupled ATP synthesis and hydrolysis catalyzed by $\mathrm{CF}_{\mathrm{o}} \mathrm{F}_{1}$-liposomes. EMBO J. 22, 418-426 (2003).

21. Okuno, D., Iino, R. \& Noji, H. Stiffness of $\gamma$ subunit of $F_{1}$-ATPase. Eur. Biophys. J. 39, 1589-1596 (2010).

22. Ueno, H. et al. Simple dark-field microscopy with nanometer spatial precision and microsecond temporal resolution. Biophys. J. 98, 2014-2023 (2010).

23. Watkins, L. P. \& Yang, H. Detection of intensity change points in time-resolved single-molecule measurements. J. Phys. Chem. B 109, 617-628 (2005).

24. Crouch, C. H. et al. Facts and artifacts in the blinking statistics of semiconductor nanocrystals. Nano Lett. 10, 1692-1698 (2010).

25. Terentyeva, T. G. et al. Dynamic disorder in single-enzyme experiments: facts and artifacts. ACS Nano 6, 346-354 (2012).

26. Taylor, W. A. Change-point analysis: a powerful new tool for detecting changes. http://www.variation.com/cpa/tech/changepoint.html (2000).

27. Slonim, N., Atwal, G. S., Tkačik, G. \& Bialek, W. Information-based clustering. Proc. Natl Acad. Sci. USA 102, 18297-18302 (2005). 
28. Taylor, J. N., Li, C.-B., Cooper, D. R., Landes, C. F. \& Komatsuzaki, T. Error-based extraction of states and energy Landscapes from experimental single-molecule time-series. Sci. Rep. 5, 9174 (2015).

29. Suzuki, T., Tanaka, K., Wakabayashi, C., Saita, E. \& Yoshida, M. Chemomechanical coupling of human mitochondrial $\mathrm{F}_{1}$-ATPase motor. Nat. Chem. Biol. 10, 930-936 (2014).

30. Watanabe, R. \& Noji, H. Chemomechanical coupling mechanism of $F_{1}$-ATPase: catalysis and torque generation. FEBS Lett. 587, 1030-1035 (2013).

31. Sumi, H. \& Marcus, R. A. Dynamical effects in electron transfer reactions. J. Chem. Phys. 84, 4894-4914 (1986).

32. Hoaglin, D. C., Mosteller, F. \& Tukey, J. W. Understanding Robust and Exploratory Data Analysis (John Wiley \& Sons, Inc., 1983).

33. Masaike, T., Koyama-Horibe, F., Oiwa, K., Yoshida, M. \& Nishizaka, T. Cooperative three-step motions in catalytic subunits of $\mathrm{F}_{1}$-ATPase correlate with $80^{\circ}$ and $40^{\circ}$ substep rotations. Nat. Struct. Mol. Biol. 15, 1326-1333 (2008).

34. Okazaki, K. \& Hummer, G. Phosphate release coupled to rotary motion of $\mathrm{F}_{1}$-ATPase. Proc. Natl Acad. Sci. USA 110, 16468-16473 (2013).

35. Watanabe, R., Koyasu, K., You, H., Tanigawara, M. \& Noji, H. Torque transmission mechanism via DELSEED loop of $\mathrm{F}_{1}$-ATPase. Biophys. J. 108, 1144-1152 (2015)

36. Abrahams, J. P., Leslie, A. G., Lutter, R. \& Walker, J. E. Structure at $2.8 \AA$ resolution of $F_{1}$-ATPase from bovine heart mitochondria. Nature 370, 621-628 (1994).

37. Ito, Y. \& Ikeguchi, M. Structural fluctuation and concerted motions in $\mathrm{F}_{1}$-ATPase: a molecular dynamics study. J. Comput. Chem. 31, 2175-2185 (2010).

38. Iino, R. \& Noji, H. Rotary catalysis of the stator ring of $F_{1}$-ATPase. Biochim Biophys. Acta 1817, 1732-1739 (2012).

39. Uchihashi, T., Iino, R., Ando, T. \& Noji, H. High-speed atomic force microscopy reveals rotary catalysis of rotorless $\mathrm{F}_{1}$-ATPase. Science 333, 755-758 (2011).

40. Efron, B. Nonparametric estimates of standard error: the jackknife, the bootstrap and other methods. Biometrika 68, 589-599 (1981).

41. Taylor, J. R. An Introduction to Error Analysis: The Study of Uncertainties in Physical Measurements 2nd edn (University Science Books, 1996).

\section{Acknowledgements}

We acknowledge H. Teramoto, J. N. Taylor, Y. Tamiya and S. Tsugawa for many helpful discussions. This work was partially supported by JSPS, Grant-in-Aid for Scientific Research (No. 25287105) (to T.K.), Grant-in-Aid for Exploratory Research (No. 25650044) (to T.K.), Grant-in-Aid for Scientific Research on Innovative Areas Spying minority in biological phenomena', MEXT (No. 23115007) (to T.K.), Grant-in-Aid for Scientific Research (No. 18074005) (to H.N.) and (No. 30540108) (to RW), MEXT and HFSP (to T.K. and C.-B.L.).

\section{Author contributions}

C.-B.L. developed the algorithm, analysed the data, wrote and prepared the manuscript. T.K. supervised the research. H.U. and R.W. designed and performed the experiments. H.N. designed the experiments and supervised the experimental research. All authors discussed the results and implications of the research and commented on the manuscript.

\section{Additional information}

Supplementary Information accompanies this paper at http://www.nature.com/ naturecommunications

Competing financial interests: The authors declare no competing financial interests.

Reprints and permission information is available online at http://npg.nature.com/ reprintsandpermissions/

How to cite this article: Li, C.-B. et al. ATP hydrolysis assists phosphate release and promotes reaction ordering in $\mathrm{F}_{1}$-ATPase. Nat. Commun. 6:10223 doi: $10.1038 /$ ncomms10223 (2015).

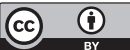

This work is licensed under a Creative Commons Attribution 4.0 International License. The images or other third party material in this article are included in the article's Creative Commons license, unless indicated otherwise in the credit line; if the material is not included under the Creative Commons license, users will need to obtain permission from the license holder to reproduce the material. To view a copy of this license, visit http://creativecommons.org/licenses/by/4.0/ 